(Aus dem deutschen physiologischen Institut zu Prag.)

\title{
Ueber secundäre Erregung von Muskel zu Muskel. Von
}

Dr. Egmont Münzer,

Assistent am physiologischen Institut der deutschen Universität in Prag.

Mit 5 Holzschnitten.

Bis in die jüngste Zeit waren Beispiele secundärer Erregung von Muskel zu Muskel trotz zahireicher darauf gerichteter Untersuchungen unbekannt.

Weder $\mathrm{H}$ erin ${ }^{1}$ ) noch $\mathrm{K}$ ii h $\mathrm{e} \mathrm{e}^{2}$ ), welche beide die Versuchsbedingungen auf's mannigfachste variirten, konnten an frischen Froschmuskeln secundäre Erregung von Muskel zu Muskel hervorrufen. Erst als $K$ it $h \mathrm{n} \mathrm{e}^{3}$ ) bei neuerlichen Versuchen die beiden zum Versuche verwendeten Muskeln an dem einen Fnde zusammenpresste, gelang es ihm, secundäre Erregung des einen Muskels durch direkte Erregung des anderen herbeizuführen. Sofort nach dem Erscheinen der Arbeit $K u ̈ h n e$ 's versuchte es Biedermann ${ }^{4}$ ), die ausgebreiteten, tetanischen Muskelcontractionen, welche bei Reizung begrenzter Muskelpartien enthäuteter, oberflächlich vertrockneter Frösche eintreten, durch eine, in Folge der ausserordentlich gesteigerten Erregbarkeit möglich gewordene secundäre Erregung zn erklären; gleichzeitig äusserte er die Meinung, dass es sich sowohl bei Kühne's als bei seinen Versuchen um die gleiche Ursache, nämlich um den Wasserverlust und den dadurch bedingten innigeren Contact der Muskelfasern handeln könne. Keinem Forscher aber war es bisher gelnngen, secundäre Erregung von Muskel zu Muskel bei Benützung ganz normaler Muskeln zu erhalten.

1) Wiener akad. Sitzungsberichte Bd. 79, III. Abth., S. 27.

2) Untersuch. aus d. Physiol. Instit. Heidelberg Bd. III, S. 83.

3) Zeitschrift f. Biologie 1888; Nene Folge Bd. VI, S. 383-422.

4) Wiener akad. Sitzungsber. Bd. 97, III. Abth., S. 154-160. 
Nun hat Regec zy eine Arbeit ${ }^{1}$ ) über dieses Thema veröffentlicht, in welcher er auf Grund zahlreicher Versuche diese Luicke ansgefïllt zu haben glaubt. War es an und für sich schon auffallend, dass es $\mathrm{Regecz}$ z. Th. unter denselben Versuchsbedingungen, bei welchen $\mathrm{Her} \mathbf{i} g$ und $K$ ïh $\mathrm{ne}$ an frischen Muskeln experimentirt hatten, gelungen sein sollte, was den genannten Forschern trotz darauf gerichteter Aufmerksamkeit nie geglïckt war, so waren es noch Bedenken anderer Art, die beim Studium der Arbeit Regeczy's zur Nachuntersuchung aufforderten, Bedenken, welche weiterhin ausführlicher zur Sprache kommen sollen. Hier sei noch kurz erwähnt, dass sich Regeczy bei seinen Untersuchungen vorzugsweise des M. rect. int. maior (Ecker) (d. i. der M. gracilis Du-Bois Reymond's) bediente, eines Muskels, der durch eine sehr schräg verlaufende, die ganze Dicke des Muskels durchsetzende Inscription in zwei Theile geschieden ist, und dass er Contractionen beider Partien nach ausschliesslicher Reizung der einen gesehen zu haben glaubt, während Prof. $\mathrm{Hering}$, wie er mir mittheilte, sich seinerzeit überzeugt hatte, dass jede streng auf die eine Muskelhälfte beschränkte Reizung auch nur diesen und nie zugleich die andere zur Contraction bringt.

Meine eigenen Versuche sind an curarisirten Fröschen (R. temporaria und esculenta) angestellt. Legt man den M. rect. int. mai. auf eine Glasplatte und reizt die eine Hälfte mit einzelnen Inductionsschlägen, indem man zwei Platindrahtelektroden von $2 \mathrm{~mm}$ gegenseitigem Abstand quer zum Faserverlauf anlegt, so beobachtet man auf das allerschönste, wie diese Muskelhälfte sich verkürzt, an der Inscription wulstig vorspringt und sich scharf von der anderen Muskelpartie abhebt. Niemals und wenn man selbst die von Regeczy geforderten sehr starken Ströme anwendet (1-3 Daniell'sche Elemente im primären Kreise, secundäre Rolle des $\mathrm{Du}-\mathrm{B}$ o is'schen Schlittenapparates mit 4115 Windungen vollständig über die primäre geschoben), beobachtet man eine Zuckung der zweiten Muskelhälfte, ausgenommen den Fall, dass man mit den Elektroden in allzugrosse Nähe der Inscription kommt. Reizt man die betreffende Muskelhälfte nicht mit einzelnen Inductionsschlägen, sondern lässt man den N e e f'schen Hammer spielen und teta-

1) Pflüger's Archiv f. Physiologie 44. Bd., S. 469-512. 
nisirt, so tritt stets nur eine Dauer-Contraction der gereizten Partie ein, ohne dass jemals eine Verkürzung der anderen beobachtet wird, auch hier den obigen Fall ausgenommen. Dass es sich aber im Falle eines Uebergreifens der Contraction anf die andere Muskelhälfte um Stromschleifen handelt, liegt auf der Hand und kann leicht dadurch bewiesen werden, dass diese zweite Muskelhälfte ebenso zuckt, wenn man die erste nahe der Inscription abschneidet, an ihre Stelle ein ähnlich geformtes Stiuck Kochsalz-Thon bringt und an letzteren die Elektroden fest anlegt. Sobald man jetzt mit den Elektroden wieder in dieselbe Nähe der Inscription kommt, zuckt auch wieder die zweite Muskelhälfte.

Reizt man den M. rectus abdominis in situ (man enthäutet einfach den geköpften Frosch) und zwar eine der mittleren Partien dieses mehrere quere Inscriptionen besitzenden Muskels, so sieht man, wie die gereizte Partie sich verkürzt, und die beiden diese Muskelpartie begrenzenden Inscriptionen einander genähert werden. Niemals aber wird man eine Verktirzung der angrenzenden Muskelpartien erhalten. Der Hinweis Regeczy's auf die Thatsache, dass es sich hier um eine quere, nicht aber um eine schräg verlaufende Inscription (wie beim M. rect. int. maior) bandelt, worauf er besonderes Gewicht legt ${ }^{1}$ ), kommt deshalb nicht in Betracht, weil wie gesagt auch an letzterem Muskel stets nur die gereizte Muskelhälfte reagirt, sofern Stromschleifen vermieden sind.

Viel überzeugender aber werden diese Versuche, wenn man sie an Muskeln von gesteigerter Erregbarkeit vornimmt. Biedermann'), der zuerst, wie oben erwähnt, derartige Versuche anstellte, hat, da keine Veranlassung hierzu vorhanden war, auf das gegenseitige Verhalten zweier durch eine Inscription getrennter Muskelpartien nicht geachtet. Präparirt man in der von ihm angegebenen Weise einen Frosch - zu diesen Versuchen wird am besten $R$. temporaria benützt - und reizt mechanisch oder mit schwächsten elektrischen Strömen die eine Hälfte des leicht oberflächlich in passendem Grade eingetrockneten M. rect. intern. maior, so sieht man auf das beste, wie die gereizte Partie in eine meistens lang anhaltende Verkürzung geräth. Hierbei wird die Inscription sebr stark gegen den Anheftungspunkt der gereizten

1) Regeczy, loc. cit. S. 483.

2) Loc. cit. 
Muskelpartie am Knochen herangezogen und die nicht gereizte Muskelhälfte gedehnt. Die contrahirte Partie runzelt und verdickt sich und ist durch die Inscription von der gedehnten Muskelhälfte scharf getrennt. Mitunter werden von der direkt gereizten Muskelhälfte aus die daneben und darunter liegenden Muskeln secundär erregt, und von diesen aus sieht man manchmal die Erregung auf die andere Muskelhälfte des inscribirten Muskels übertragen werden. Bertihrte ich, während die gereizte Muskelhälfte verkürzt war, die andere gedehnte, so trachtete diese sich zu verkürzen, die Inscription gegen sich heranzuziehen und nun brach ein Kampf um die Inscription zwischen beiden Partien aus, es kam zu einem Hin- und Herwogen zwischen beiden Muskelhälften, welches allmählich abklang oder plötzlich mit Erschlaffung beider Muskeln endigte.

Noch viel schöner gelingen diese Versuche am M. rect. abdominis. Berührte ich die unterste an das Becken inserirte Partie, so verkïrzte sich stets diese allein und zog den ganzen übrigen Muskel an sich heran. Wurde eine der mittleren Muskelpartien mechanisch gereizt, so contrahirte sich diese und dehnte vorzliglich die beiden benachbarten Muskelfelder.

Diese eben angeführten Versuche sprechen schon auf das entschiedenste gegen eine secundäre Erregung von Muskel zu Muskel durch eine Inscription bindurch. Trotz der ausserordentlich gesteigerten Erregbarkeit der zu den Versuchen benutzten Muskeln waren wir nicht im Stande eine sekundäre Erregung durch eine Inscription hindurch zu beobachten. Dennoch unternahm ich es, die Regeczy'schen Untersuchungen in einer der seinen analogen Untersuchungsweise zu wiederholen. Der Apparat (Fig. 1), dessen ich mich hierbei bediente, und der ebenfalls Doppelschreibung gestattete, befand sich bereits seit längerer Zeit im hiesigen physiologischen Institute, und da er auch zu anderen Untersuchungen gut verwendbar ist, sei er hier ausführlicher beschrieben.

Auf einem Metallfusse befindet sich eine Triebstange, auf welcher zwei Triebkasten mittels der Knöpfe $k$ vertical verschiebbar sind. Die mit den Triebkasten fest verbundenen horizontalen Arme $(a)$ bestehen aus 2 Theilen, die durch eine Schraube in horizontaler Ebene gegen einander verschoben werden können, wodurch die Spitzen der Zeichenhebel mit der Schreibfläche in sicheren Contact gebracht werden können. Iie Verbindung der Zeichenhebel $(z)$ mit den Armen ist so hergestellt, dass diese Arme an ihren vorderen Enden stellbare verticale Axen tragen, welche sich nach aufwärts gabelig theilen, 
und die beiden Bläter der Gabel Träger der horizontalen leicht beweglichen Axen sind, um welche sich die aus Strohhalmen gefertigten Zeichenhebel bewegen. Mittels der an ihrem rückwärtigen Ende befindlichen (Doppel-) Spitzen können die Zeichenhebel in den Muskel eingestochen werden; das vordere Ende derselben ist mit einer feinen, aber stumpfen Drahtspitze montirt. Um den Muskel fixiren zu können, befindet sich am oberen Ende der Triebstange eine Stellschraube (s), mittels deren ein Messingschieber in verschiedener Stellung befestigt werden kann. Dieser Messingschieber trägt an seinem freien Ende eine Hartgummiklemme zum fixiren des Knochens ${ }^{1}$ ). Am unteren Ende der Triebstange schiebt sich ohne Trieb eine Zwinge, die einen Ring $(r)$ trägt, in welchen man ein Gefäss mit Flüssigkeit setzen kann zur Dämpfung der Schwingungen des die

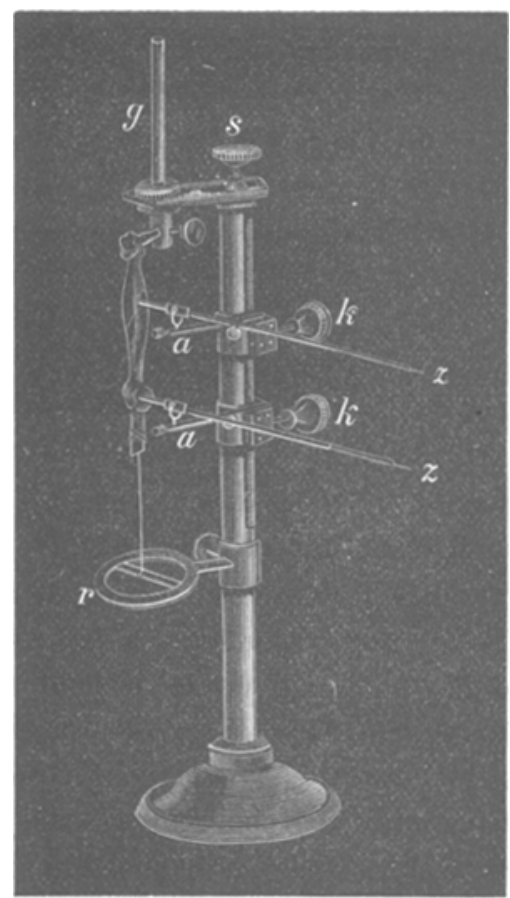

Fig. 1.

Spannung des Muskels bewirkenden Gewichtes ${ }^{2}$ ) oder der Ring dient indirekt mittels eines eingekeilten Stück Holzes zur Befestigung eines an Stelle des Gewichtes verwendeten elastischen Fadens, wie die Figur 1 es zeigt.

Bei den mit diesem Apparate angestellten Versuchen wurde folgendermaassen vorgegangen: Der frei präparirte, aber beiderseits im Zusammenhange mit den Knochen gelassene M. rect. int. maior eines curarisirten Frosches wurde mit der 'Tibia in der oberen Klemme befestigt; der obere Zeichenhebel in die obere Muskelpartie dicht iiber der sehnigen Inscription, der untere Hebel in die knorpelige Beckenpfanne eingestochen. Zur Reizung dienten

1) Die Hartgummiklemme ist mit einem Glasrohr (g) montirt, so dass sie unter Umständen auch als unpolarisirbare Elektrode verwendet werden kann, für den Fall, dass man den Strom am natürlichen Ende des Muskels ein-, resp. austreten lassen will.

2) Es ist selbstverständlich, dass die im Gefässe vorhandene Flüssigkeit die zweite Elektrode bilden kann, wobei dann der Strom auch hier am natür lichen Muskelende ein-, resp. austritt. 
einzelne Inductionsschläge von derselben Stärke, wie in den eingangs erwähnten Versuchen; anch die Platindrahtelektroden waren dieselben und wurden stets quer uiber den Muskel gelegt.

Betrachten wir nun die auf diese Weise gewonnene Curve Fig. 2, welche vom $R$. int. mai. einer $R$. temporaria geschrieben

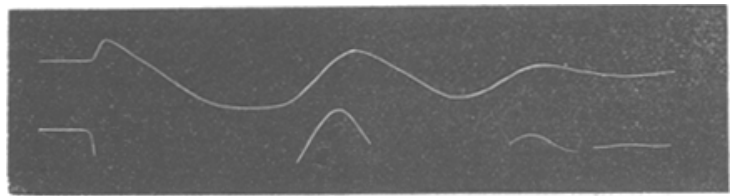

Fig. 2.

wurde. Der Muskel war am unteren Ende mit $5 \mathrm{gr}$ belastet, welche in gewöhnliches Wasser als Dämpfungsflissigkeit tauchten. Im primären Kreise befand sich ein Daniell'sches Element; der zur Reizung benutzte Oeffnungsinduktionsschlag wurde ausgelöst durch Oeffnung eines Hg-Schlïssels. Die Zeichenhebel schrieben in diesem Fall auf einer mit der Hand gedrehten, berussten Trommel. Die Krümmung der Cylinderfläche brachte es mit sich, dass vom unteren Hebel nur die der Abscisse nächsten Theile der Curve gezeichnet wurden; von wesentlicher Bedeutung ist jedoch nur die vom oberen Zeichenhebel gezeichnete Curve. Der obere Zeichenhebel verzeichnete nach einer anfänglichen Dehnung, welcher der kurze aufsteigende Theil der Curve entspricht, eine kleine Verkiirzung, welche sich durch ein Herabgehen der Curve unter die Abseisse verräth. Doch wir sehen, dass der Muskel nochmals gedehnt wird, sich nochmals verkürzt; es handelt sich eben um Longitudinal-Schwingungen des Muskels und also auch seiner oberen Hälfte. Durch die Zuckung der u n t e re n Muskelhälfte wird das anhängende Gewicht hinaufgeschleudert und der durch das Gewicht gedehnt gewesene Muskel wird dabei vermöge seiner Elastizität kürzer, als er geworden wäre, wenn z. B. die Beschleunigungen des bewegten Gewichts durch passende Reibungswiderstände aufgehoben worden wären. Bei den folgenden Versuchen wurde ein Gewicht von 8-10 gr zur Belastung genommen, an der Unterfläche des Gewichtes ein Scheibchen steifen Papiers mittels Siegellacks befestigt und als dämpfende Flüssigkeit dickes Glycerin genommen. Um vollständig ausgeschriebene Curven zu erhalten, benützte ich bei allen weiteren Versuchen als Schreibfläche eine ebene Russfläche. Das im hiesigen physiologischen Institute 
befindliche Kymographion ,ist mit einer Einrichtung versehen, durch welche das Papier mit hinreichender Spannung iiber zwei, $10 \mathrm{~cm}$ von einander abstehende dünne Verticalwalzen gezogen wird, so dass es zwischen denselben eine schmale ebene Fläche bildet" ${ }^{1}$ ). Ein Beispiel der auf diese Weise gewonnenen Curve zeigt Fig. 3

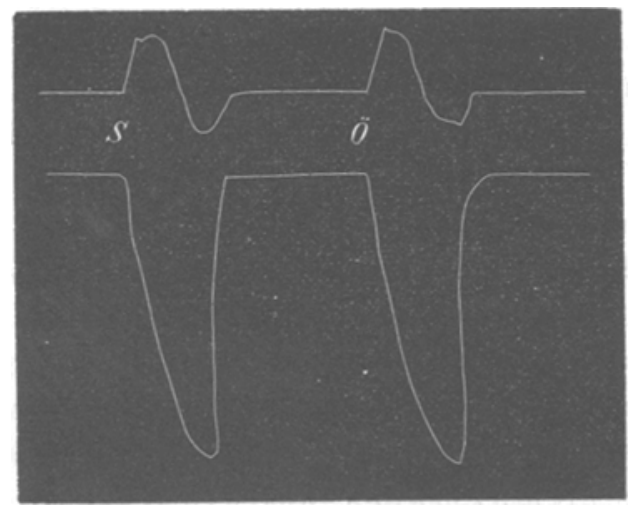

Fig. 3.

und zwar ist die erste $(s)$ durch die Schliessungs-, die zweite $(\ddot{\partial})$ durch den Oeffnungs-Induktionsschlag erzengt. Mit dern Beginn der Verkuirzung der direkt gereizten unteren beginnt die Dehnung der oberen Muskelhälfte; das dadurch bedingte Ansteigen der oberen Curve geht aber bald in ein Absteigen bis unter die Abscisse ïber, was einem Ktirzerwerden der oberen Muskelhälfte entspricht. Ist also die obere Muskelpartie von Seiten der direkt gereizten, unteren secundär erregt worden? Keineswegs! Die Ursache der scheinbaren Verkïrzung des oberen Muskels liegt abermals in der Verwendung eines Gewichtes zur Belastung. Sobald nämlich der untere Muskel sich contrahirt, zieht er seine beiden Enden gegen einander, d. h. er hebt einerseits das Gewicht, dehnt andererseits die obere Muskelhälfte. Nun aber fliegt das einmal in Bewegung gesetzte Gewicht vermöge seiner Trägheit über die eigentliche Hubhöhe hinaus („Wurfhöhe"). Im selben Augenblicke wird der ganze Muskel, also auch die obere Partie entlastet; letztere schnellt daher zusammen, sie verkürzt sich, und es täuscht dies eine vitale Contraction vor.

1) Hering: Ueber die Methoden zur Untersuchung u. s. w. Wiener Akademieberichte 79. Bd., III. Abth., Aprilheft S. 261 (Sep.-Abd. S. 25). 
Man könnte gegen die hier gegebene Erklärung vielleicht einwenden wollen, dass es sich um wirkliche secundäre Erregung handle, wenn wir nicht im Stande gewesen wären, den vollständigen Beweis für die eben gegebene Erklïrung zu liefern.

Wir behaupteten, dass die Ursache der s ch e inbar en a ctiven Verkürzung der oberen Muskelpartic in der Auwendung eines Gewichtes als des vom Muskel zu uiberwindenden Widerstandes zu suchen sei, und es handelte sich also lediglich darum, den thätigen Muskel einen zweckmässiger gewählten Widerstand iberwinden zu lassen. Dies geschah einfach durch Anwendung eines mässig gespannten Gummifadens. Derselbe wurde einerseits an den Hiiftknochen des Präparates angebunden, andererseits an einem Holzstäbchen befestigt, das in den Metallring (r) des Apparates eingekeilt wurde, aus welchem das Gefäss mit Flüssigkeit entfernt worden war. Nun wurde dieser Ring so gestellt, dass das Gummiband leicht gespannt war. Reizte man dann die untere Muskelhälfte, so gelang es niemals auch nur die leiseste Andeutung einer Verkürzung der oberen Muskelpartie zu beobachten, ausgenommen den Fall, dass man mit den Elektroden bis in die Gegend der. Inseription selbst kam. Man kann selbst sehr starke Ströme anwenden (3 Daniell's im primären Kreise), stets wird entsprechend der Verkürzung der unteren direkt gereizten Muskelhälfte die obere einfach gedehnt, so lange eben die untere verkürzt ist.

Zur Illustration des Gesagten möge Fig. 4 dienen. Es ent-

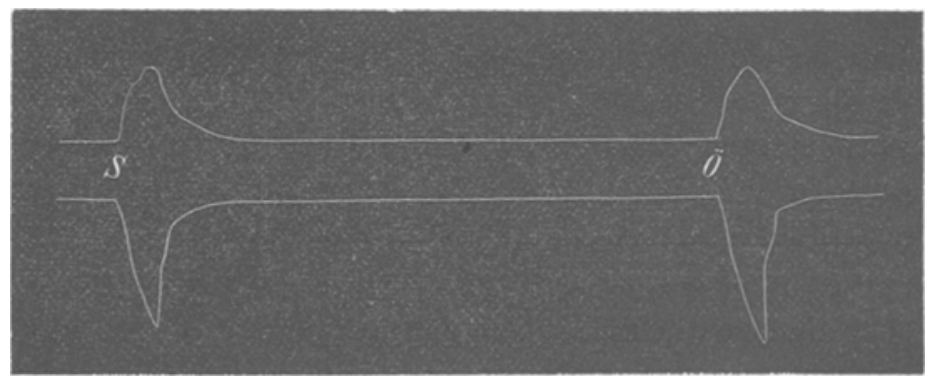

Fig. 4.

stammt diese Curve dem R. intern. maj. eines sehr kräftigen Exemplars von R. esculenta. Der frei herabhängende Muskel war $43 \frac{1}{2} \mathrm{~mm}$ lang, mit Gummispannung betrug seine Länge $47 \mathrm{~mm}$. Die Länge der Muskelpartic oberhalb des oberen Zeichenhebels 
war $19 \mathrm{~mm}$. In diesem Falle befanden sich im primären Kreise 3 Daniell'sche Elemente, die Rollen waren iiber einander geschoben und die Platindrähte, welche als Elektroden dienten, waren wieder $2 \mathrm{~mm}$ von einander entfernt quer iuber die Mitte der unteren Muskelhälfte gelegt. Man erkennt nun an der Figur klar die oben auseinandergesetzten Verbältnisse: die Dehnung der oberen Muskelhälfte so lange dauernd, als die untere verkurzt is t. Kommt man freilich mit den Elektroden in allzugrosse Nähe der Inscription, dann ändert sich das Bild sofort, wenn die obere Muskelpartie miterregt wird und gestaltet sich so, wie es Fig. 5 zeigt.

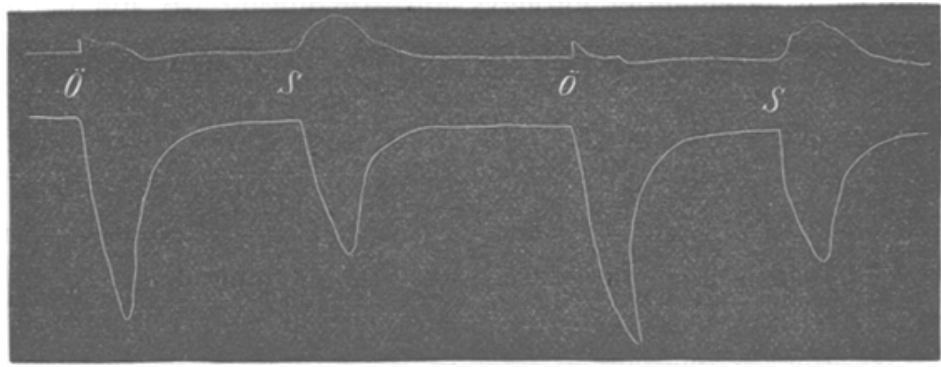

Fig. 5.

Wir sehen an der durch den Oeffnungsinduktionsschlag ausgelösten Curve (ö) die anfängliche Dehnung der oberen Muskelhälfte, welcher der kurze aufsteigende Theil der oberen Curve entspricht, sofort coupirt werden, und an deren Stelle eine unregelmässige, selbst ein wenig unter die Abscisse hinunter gehende Linie treten, was einer aktiven Contraction dieser Partie entspricht. Die Stromschleifen des schwächer wirkenden Schliessungsinduktionsschlages sind dagegen zu schwach, um eine Erregung der oberen Muskelhälfte auszulösen; sie wird einfach gedehnt $(s)$.

$O b$ nun in den von $R$ egeczy auf ähnliche Weise angestellten Versuchen (S. 493-501) wirklich der durch Anwendung eines frei herabhängenden Gewichtes herbeigeführte Fehler übersehen wurde, wage ich nicht zu behaupten. Möglich wäre dies wohl, da auch nach seinen Zeichnungen das zur Belastung verwendete Gewicht - dessen Grösse er übrigens bei diesen Versuchen ebensowenig wie die genaue Reizgrösse angiebt - frei herabgehangen zu haben scheint.

Reg e c z y hat aber noch auf andere Weise die sekundäre E. Pfüger, Archiv fūr Physiologie. Bd. XIVI. 
Erregung von Muskel zu Muskel nachzuweisen gresucht. Ich habe einen Theil der Versuche, wie ich jetzt vorausschicken darf, mit ebenso negativem Erfolge, als die bereits angeführten, wiederbolt. Was zuerst die nach der Bezold'schen Methode angestellten Versuche betrifft, sagt R e g e ezy S. 483: „Ich konnte nämlich nach der Bezold'schen Methode die obere Hälfte des Muskels mittels zweier Zangen unbeweglich machen, so dass das Myographium nur die Bewegung der unteren Muskelhälfte aufzeichnete". Hering macht nun in seiner wiederbolt erwähnten Arbeit „über die Methoden zur Untersuchung der polaren Wirkungen des elektrischen Stromes im quergestreiften Muskel" S. 22 (258) ausdrücklich auf die starke Einklemmung aufmerksam, die nöthig ist, um bei derartigen Versuchen die obere Muskelpartie unbeweglich zu machen, Wenn man einen frei herabhängenden oder an seinem unteren Ende belasteten Muskel in der Mitte abklemmt und die obere Partie reizt, wird man sich leicht überzeugen können, dass die obere Muskelhälfte die untere etwas durch die Klemme hindurch hinaufzieht, so lange nicht der Druck der Klemme ausserordentlich gross ist, so lange es also nicht zu einer sehr starken Quetschung der Muskelpartie gekommen ist. Schon aus diesem Grunde kann man diesen Versuchen gegenüber nicht skeptisch genug sein, um so mehr, wenn es sich nicht blos um den Sartorius, sondern um einen so dicken Muskel handelt, wie es der R. int. maj. ist.

$R$ e ge c z y hat ferner den $R$. int. maj. mit seiner sehnigen Inscription auf ein Stiutzstäbchen gelegt, denselben mittelst zweier durch die Gegend der Inscription hindurch gesteckten Stecknadeln fixirt und die beiden frei herabhängenden Muskelenden mit den Zeichenhebeln verbunden (ob und wie stark er die beiden Enden belastet hat, giebt er nicht an). Reizte er nun das eine Ende des Muskels, so beobachtete er eine etwas verspätete Verklirzung des zweiten Endes. Wiederholt man diesen Versuch auf dieselbe Weise, so kann man allerdings mitunter eine äusserst schwache Bewegung dieses Endes beobachten, welche sich jedoch sehr leicht erklären lässt. Reizen wir nämlich die eine Muskelhälfte, so contrahirt sich diese, und da wegen des sehr schrägen Verlaufs der Inscription ein kleiner Theil ihrer Fasern auch tiber die fixirenden Nadeln in's Bereich der andern Muskelhälfte hinüberreicht, so kommt es zu einer, aber äusserst geringen Bewegung dieser letzteren. Eine wirkliche Hebung der einen Partie nach 
Reizung der anderen zu constatiren, war ich jedoch nie im Stande ${ }^{1}$.

1) Bei der Besprechung dieser Versuchsmethode hat Regeczy S. 502 einen Irrthum begangen, den richtig zu stellen ich mir mit Genehmigung meines verehrten Lehrers, Herrn Prof. Hering, hier erlaube.

Regeczy schreibt in seiner Arbeit über secundäre Erregung S.502: „Eine zu dieser Art der Lagerung - es sind jene Versuche gemeint, wo der Muskel mit seiner Mitte auf einem Stützstäbchen fixirt war - ähnliche Versuchsanordnung wurde schon von A eby*) zur myographischen Untersuchung der polaren Reizwirkung des constanten elektrischen Stromes verwendet; nämlich bei seinen späteren Versuchen. Der Muskel hielt bei diesen Versuchen eine Zange in der Mitte schwach abgepresst (?); aber zum myographischen Zeichnen diente nur die eine, entweder die obere oder die untere Muskelhälfte; in einigen Versuchen vertauschte $\mathbf{A}$ e by die Zange mit einer den Muskel in der Mitte durchbohrenden Nadel, um der Zusammenpressung des Muskels in der Länge der intrapolaren Strecke auszuweichen. - Die Einrichtungsweise dieser letzteren Versuche ist bei Hering**) unrichtig wiedergegeben, nämlich so: "Der Muskel wurde am oberen Ende befestigt und quer durch seine Mitte eine Nadel geführt, welche mit dem zeichnenden Stifte in Verbindung stand" "; - es diente aber zur Fixirung offenbar die Nadel, anstatt einer Zange, und der Zeichenhebel wurde durch das eine Muskelende in Bewegung gesetzt."

Bei der Durchsicht dieser Sätze fällt auf, dass die von $R$ e g e c z y gegebene Darstellung der A eby'schen Versuchsanordnung in ihrem ersten Theile mit der $\mathrm{H}$ ex ing'schen ***) übereinstimmt. Mit der Behauptung jedoch, dass bei den weiteren Versuchen A eby's die Nadel zur Fixirung des Muskels dienen sollte, befindet sich Regeczy im Irrthume. Nachdem Aeby die anfünglichen Versuche so angestellt hatte, dass der Muskel in der Mitte eingeklemmt war, wodurch „die Verschmelzung der Zuckung der einen Hälfte mit derjenigen der anderen verhindert wurde" $\dagger$ ), wollte er auch noch den Beweis liefern, „dass dieser Umstand (nämlich die Einklemmung) in keiner Weise an der Natur der gefundenen Resultate betheiligt sei". "Ich stellte deshalb", sagt Aeby, "eine Anzahl von Versuchen an, bei denen ich die Einklemmung vermied, indem ich den zeichnenden Apparat an einer quer durch die Mitte des Muskels gestochenen Nadel aufhing, während alles Uebrige unverändert blieb. Bei dieser Anordnung konnte ebenfalls nur die eine Muskelhälfte, und zwar die obere wirken, während die

*) A e by, Reichert's u. Du-Bois Reymond's Archiv 1867, S. 699.

**) Loc. cit. S. 255 (Sep.-Abdr. S. 19).

***) Loc. cit. S. 254 (Vierte Gruppe).

f) A e b y, loc. cit. S. 695 . 
R e g e c zy behauptet endlich weiterhin, nach seinen Versuchen secundäre Erregung von Muskel zu Muskel an zwei Muskeln gesehen zu baben, die nur an einer kurzen Strecke ihrer Länge an einander gelegt und in ihrer gegenseitigen Lage durch Stecknadeln fixirt waren. Diese Versuche zu wiederholen fand ich mich nicht bemiissigt, nachdem alle vorangehenden Versuche negativ ausgefallen waren, Versuche der letzteren Art iibrigens von vielen Forschern zu wiederholten Malen mit negativem Resultate ausgefuihrt wurden, ausgenommen den Fall, dass die beiden Muskeln an dieser Stelle fest an einander gepresst werden, was in den $\mathrm{Re}$ ge ezy'schen Versuchen nach seinen Angaben nicht statt hatte, in welchem Falle übrigens diese Versuche nur Wiederholungen der $\mathrm{K} \mathfrak{u} \mathrm{h} \mathrm{n}$ e'schen gelungenen Versuche gewesen wären.

Ich glaube mich nach dem Mitgetheilten zur Behauptung berechtigt:

Eine secundäre Erregung von Muskel zu Muskel durch eine Inscription hindurch ist von $\mathrm{Re} g \mathrm{ecz} y$ nicht erwiesen worden.

\section{Anhang.}

Es sei hier gestattet auf einen fundamentalen Versuchsfehler $R$ e geczy's in einer anderen Arbeit hinzuweisen. $R$. hat im 43. B. des Pfluiger'schen Archivs für Physiologie eine Abhandlung veröffentlicht, „über die Bestimmung der Entstehungsstelle der Erregung im Muskel etc." und kommt in derselben auch auf die von B i ed ermann mit dem Hering'schen Doppelmyograph gemachten Untersuchungen zu sprechen. Den beiden auf S. 554 vorhandenen Abbildungen nach $\mathrm{zu}$ schliessen, hat er die beiden Elektroden direkt dem Muskel angelegt und schreibt auch wörtlich (S. 11): „Beide Versuchsanordnungen (seine u. Biedermann's) haben gemein, dass die zeichnende Muskelhälfte mit der einen Elektrode unmittelbar in Berïhrung steht." Das ist eine irrige Meinung.

andere vollkommen ausgeschlossen war, ohne dass die einzelnen Muskelfasern die geringste Beschädigung erlitten hätten".

Beim Vergleiche des von A e by gesagten und des von $\mathrm{Her}$ ing darüber gesohriebenen, wird jeder sehen, dass $\mathrm{H}$ ering die Stelle richtig, R e$\mathrm{g}$ e c z y jedoch sie missverstanden hat. 
H e r in g ${ }^{1}$ ) hat sein Doppelmyograph so construirt, dass der Strom dem Muskel beiderseits durch Knochen und Sehnen zugeleitet wird und dass die Klemmen, in welchen die Knochen befestigt werden, zugleich als unpolarisirbare Elektroden bentitat werden können. Hering kannte eben sehr genau den wesentlichen Unterschied, der durch eine andere, etwa seitliche Anlagerung der Elektroden an den Muskel herbeigefuhrt wird. Es ist sehr leicht zu zeigen, welchen wesentlich verändernden Einfluss auf den Verlauf der Stromfäden und die daraus resultirenden Ein- und Austrittstellen des Stromes in den einzelnen Muskelfasern eine derartige seitliche Anlagerung der Elektroden an den Muskel hat. Folgender von H. Prof. $\mathrm{Hering}$ angegebener Versuch möge das Gesagte beweisen: Man präparirt beide Sartorii eines curarisirten Frosches in der Art, dass man die beiden Muskeln an der Symphyse zusammenhängen lässt und die beiden Unterschenkelknochen die freien Enden des Präparates bilden. Die beiden Tibiae werden nun in zwei Muskelhalter geklemmt, das ganze Präparat horizontal leicht ausgespannt und die Symphyse locker gestützt. Leitet man nun mittels unpolarisirbarer Elektroden, welche an die beiden Unterschenkelknochen angelegt werden, einen starken constanten Strom (3-5 Daniell'sche Elemente) durch die beiden Sartorii und lässt während der Strom durch das Präparat hindurch fliesst einen Tropfen 0,6\% ClNaLösung auf einen der beiden Muskeln auffallen oder berührt einen der Muskeln mit einem Pinsel, welcher mit derselben Flüssigkeit voll getränkt ist, so zuckt immer derjenige Muskel, welchen der Tropfen oder der Pinsel berührte; niemals verkürzt sich der zweite Sartorius ebenfalls. Es beruht diese Zuckung nicht etwa auf der Stomschwankung, herbeigeführt durch die Verbreiterung der Strombahn an der betropften oder berübrten Stelle, wie man wohl im ersten Momente glauben möchte. Denn diese Stromschwankung würde sich auch am zweiten Muskel durch eine Zuckung desselben zu erkennen geben. Die Zuckung beruht vielmehr darauf, dass der auffallende Tropfen sofort einen Stromzweig in sich aufnimmt, so dass sich neue Eintritt- und Austrittstellen des Stromes in der Muskelsubstanz bilden; es werden kathodische Stellen geschaffen und auf diesen polaren Reiz reagirt der Muskel. Dass nicht etwa ein mechanischer oder chemischer Reiz in's Spiel kommt, lässt

1) H ering, Wiener Akademieber. Bd. 79. 
Egmond Münzer: Ueber secundäre Erregung von Muskel zu Muskel.

sich dadurch zeigen, dass man den Tropfen in ganz gleicher Weise auf den undurchströmten Muskel bringen oder mit dem trockenen Pinsel iiber den durchströmten Muskel fahren kann, ohne eine Zuckung auszulösen.

Legt man, wie Regeczy, die Elektroden an die Oberfläche des Muskels, so laufen in der Nähe der Berïhrungspunkte die Stromfäden nicht blos längs, sondern theilweise quer oder schräg durch die Muskelfasern; folglich finden sich auch in der dicht unter der Anode liegenden Muskelsubstanz nicht blos Eintritt-, sondern auch Austrittstellen des Stromes aus der contractilen Substanz der Einzelfaser und es erscheint selbstverständlich, dass dann bei Anwendung starker Inductionsströme auch von der Stelle der angelegten Anode eine Erregung ausgehen kann. Klemmt man ferner, wie dies Regeczy ebenfalls gethan hat, den durchströmten Muskel in der Mitte fest, so verbiegt man hier nothwendig seine Fasern sehr stark und es ist ganz unmöglich zu verhiiten, dass sich an der gedriickten Stelle nicht Austrittstellen der Stromfäden aus der contractilen Substanz der Einzelfasern bilden und dass nicht auch hier eine direkte Erregung herbeigeführt wird. $\mathrm{He-}$ ring's Doppelmyograph bietet ja gerade den Vortheil, dass der Muskel in seiner Mitte nicht irgend wesentlich geklemmt zu werden braucht, sondern dass hier die blosse Reibung genügt ihn festzuhalten, während beide Muskelenden frei beweglich sind, nicht wie bei Regeczy's Versuchen nur die eine. B i edermann's Versuchsanordnung war also eine sehr wesentlich andere als die Regeczy's. Endlich hat $R$ e geczy sehr starke Ströme verwendet, von denen Hering S. 8 sagt: „Die im letzten Abschnitt erörterten Fehlerquellen werden besonders dann in Betracht kommen, wenn der Strom stark ist." Deshalb vermied auch Biedermann aus guten Gründen die Anwendung zu starker Ströme. Ob Inductionsströme bei hinreichend grosser stärke auch eine anodische, der Oeffnungserregung der Kettenströme analoge Erregung herbeiführen können, ist eine noch offene Frage. Die Versuche R e geczy's sind jedenfalls nicht geeignet, eine anodische Erregung der Inductionsströme zu beweisen. 